\title{
Approaches to Quantifying the Dynamics of Nanostructures using in situ Electron Microscopy
}

Thomas W. Hansen ${ }^{1}$, Pei Liu ${ }^{1}$, Jacob Madsen ${ }^{2}$, Monia Runge Nielsen ${ }^{1}$, Jakob Schiøtz ${ }^{2}$, Jakob B. Wagner $^{1}$

1. Center for Electron Nanoscopy, Technical University of Denmark, Kgs. Lyngby, Denmark.

2. Department of Physics, Technical University of Denmark, Kgs. Lyngby, Denmark.

Surfaces of nanoparticles represents a topic of interest in several fields. For example, the activity of heterogeneous catalysts strongly depends on the structure of these surfaces. The nature of these surfaces is a function of the environment to which the nanoparticle is exposed, i.e. surrounding gases and temperature. Detailed quantitative information of the surfaces under operating conditions is inherently difficult to access due to limitations of characterization equipment. Advances in electron microscopy such as MEMS heaters, reaction cells and fast cameras have provided new possibilities for in situ characterization at the atomic scale [1] giving access to even more detailed information. Knowledge of the imaging conditions is a prerequisite in order to extract meaningful results. Application of automated analysis can provide strong and robust characterization of nanoparticle structure and dynamics can be achieved. However, automated image analysis does have its pitfalls. Whereas this is also true for manual analysis, at least the human factor can be ruled out.

In the present study, we investigate the surface structure and dynamics of gold nanoparticles under varying atmospheres. Using environmental high-resolution transmission electron microscopy, gold nanoparticles supported on cerium dioxide have been imaged under varying conditions.

Firstly, the equilibrium shape under oxidizing and reducing conditions was investigated. Under vacuum, the surfaces appeared stable meaning that no change in column occupancy, see Figure 1. The figure shows both the occupancy degree of each column as well as the diffusion frequency. As the samples are exposed to oxygen at $4.5 \mathrm{~Pa}$, the atomic columns on both $\{111\}$ and $\{001\}$ facets start diffusing. When the samples are exposed to hydrogen, only the atomic columns on the $\{001\}$ facets diffuse. These observations indicate that as expected, different gas molecules interact differently with different facets. A possible explanation of the observed events is the higher desorption temperature of oxygen $(>415 \mathrm{~K})$ compared to hydrogen $(\sim 120 \mathrm{~K})$.

Under exposure to $4.5 \mathrm{~Pa}$ carbon monoxide and $300^{\circ} \mathrm{C}$, surface layers start shifting in a concerted fashion, and a crystal twin parallel to the nanoparticle/support interface forms and moves dynamically resulting in at least two distinct configurations of the nanoparticle. In each configuration, the surface strain on the nanoparticle was measured using a newly developed routine, see Fig. 2 [2]. In the untwinned configuration (upper pane), the lower (111) facet shows strong outwards relaxation. In the twinned configuration (lower pane), the corner sites show an outward relaxation. These observations strongly suggest that the catalytically active sites are dynamic rather than static entities.

\section{References:}

[1] T.W. Hansen and J.B. Wagner, eds. "Controlled Atmosphere Transmission Electron Microscopy”. 1 ed. 2016, Springer. 
[2] J. Madsen et al, Advanced Structural and Chemical Imaging 3 (2017), p. 12.
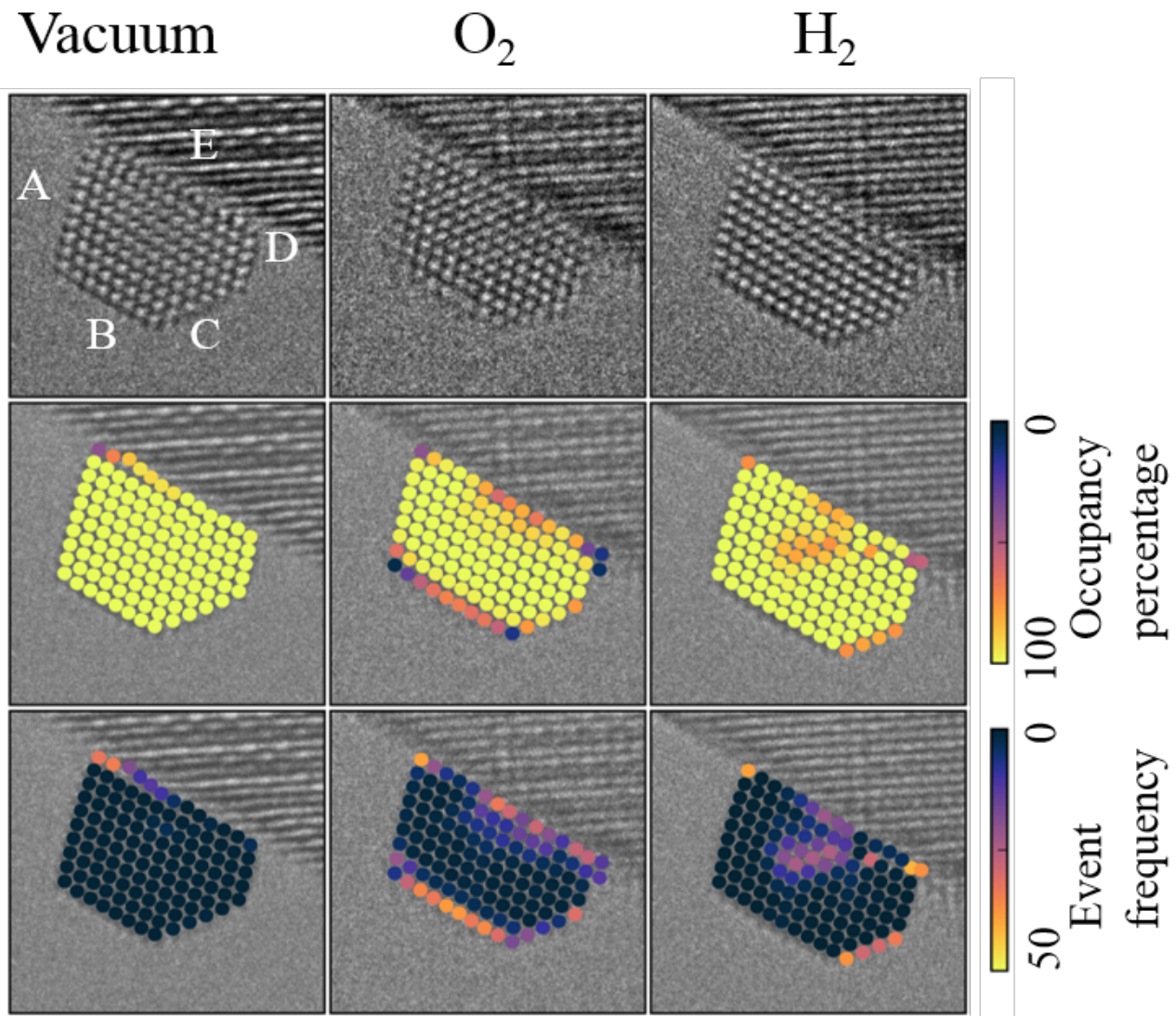

Figure 1: Quantification of occupancy and hopping frequency on the surface of a gold nanoparticle at room temperature.

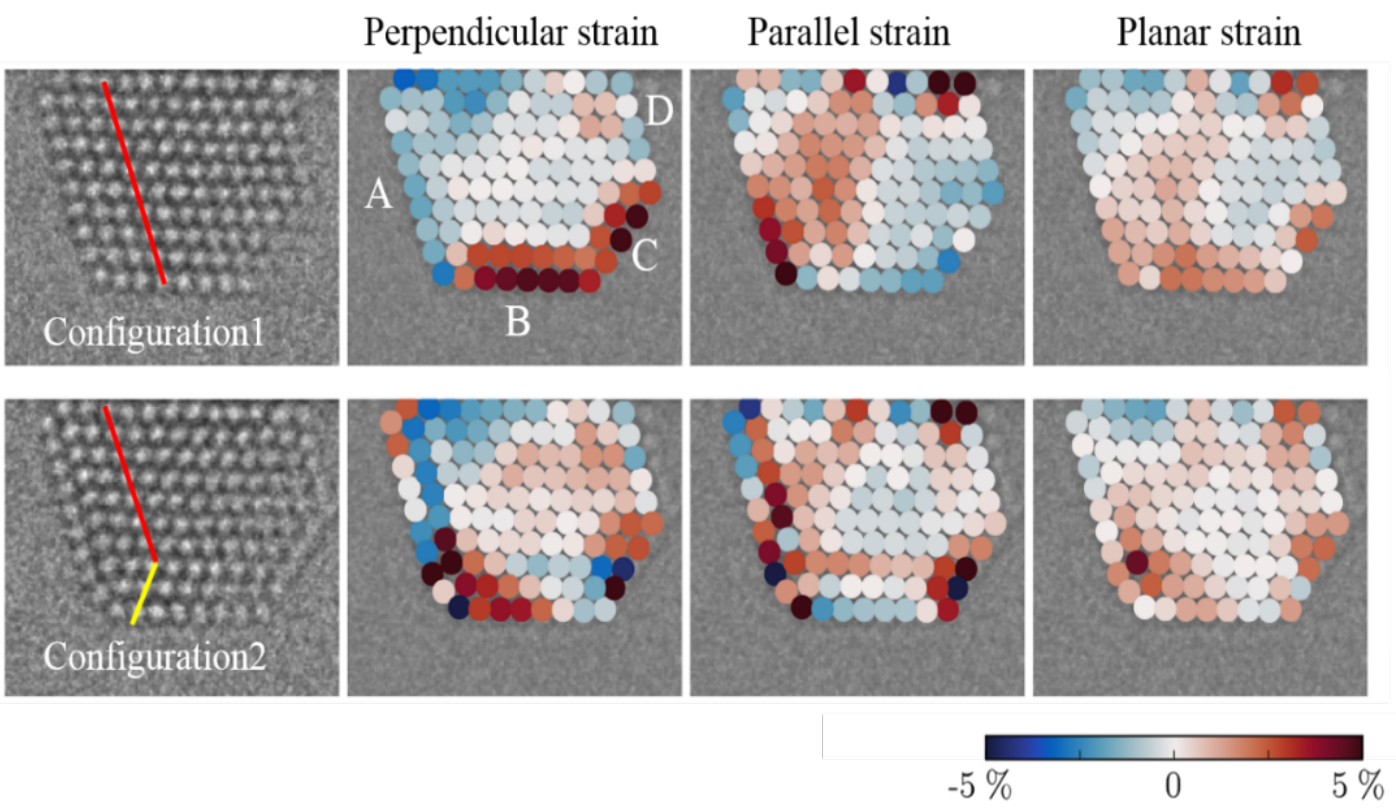

Figure 2: Surface strain on a gold nanoparticle in $\mathrm{CO}$ at $300^{\circ} \mathrm{C}$. 